\title{
INFLUÊNCIA DA INCORPORAÇÃO DE VINHAÇA NAS PROPRIEDADES DE COMPÓSITOS CIMENTÍCIOS NO ESTADO FRESCO
}

\section{INFLUENCE OF THE INCORPORATION OF VINASSE ON THE PROPERTIES OF CEMENTITIOUS COMPOSITES ON FRESH STATE}

Victor Nehring, Rayssa Silva Menezes, Lucas Henrique Pereira Silva, Jacqueline Roberta Tamashiro Berguerand Xavier, Fabio Friol Guedes de Paiva, AngelaMitie Otta Kinoshita

Universidade do Oeste Paulista - UNOESTE, Presidente Prudente, SP.

E-mail: lucashpsilva@outlook.com.

RESUMO - Na utilização do Cimento Portland em grandes volumes de concreto, tensões de tração e compressão são introduzidas no sistema que podem apresentar patologias como microfissuras relacionadas a variações bruscas de volume e temperatura. Estudos tratam do uso da sacarose como aditivo retardador da hidratação do cimento e apresentam bons resultados. Neste sentido a vinhaça é uma fonte de sacarose ainda pouco explorada. A vinhaça é um subproduto da indústria sucroalcooleira, produzida em grandes volumes, tornando-a bastante atrativa nessa aplicação. Este estudo buscou analisar a influência da vinhaça na hidratação de pastas de cimento. Para isso a vinhaça foi caracterizada em relação ao teor de açúcares redutores totais (ART) e açúcares solúveis totais (AST) pelo método Fehling e utilizada em substituição a água nas quantidades de $25 \%$, 50\% e $100 \%$. As pastas produzidas foram analisadas a partir da consistência, tempo de pega e variação de temperatura na hidratação. Os resultados apontam que a menor quantidade de substituição (25\%) acarreta aumento nos tempos iniciais e finais de pega enquanto quantidades maiores não causaram os mesmos benefícios. O teste de calorimetria confirmou os resultados obtidos pelo ensaio do tempo de pega mostrando menor variação de temperatura da pasta com $25 \%$ de substituição da água pela vinhaça.

Palavras-chave: tempo de pega; vinhaça, calorimetria.

ABSTRACT - Tension and compression stresses are introduced in the concrete when Portland Cement are used in large volumes, inducing pathologies such as micro-cracks related to sudden changes in temperature. Sucrose is used as a cement hydration retarding additive and produce good results. In this sense, vinasse is a source of sucrose still little explored. Vinasse is a by-product of the sugar and alcohol industry, produced in large volumes, making it very attractive in this 
application. This study investigates the influence of vinasse on hydration in cement pastes. The total reducing sugars (TRS) and total soluble sugars (AST) as well as the sucrose present in vinasse was determined through Fehling method. The amount of $25 \%, 50 \%$ and $100 \%$ of vinasse were used to substitute water in cement pastes composition. The consistency, initial and final setting time and thermal variation during hydration of pastes were. The results show that the lower amount of substitution (25\%) leads to an increase in the initial and final setting time, while larger amounts did not cause the same benefits. The calorimetry test confirmed the results obtained by the setting time test, showing less heat of hydration of the paste with $25 \%$ substitution of water for vinasse.

Keywords: setting time; vinasse; calorimetry.

\section{INTRODUÇÃO}

O cimento Portland é um aglomerante hidráulico cuja produção se dá pela mistura exata entre argilas, composta majoritariamente de $\mathrm{SiO} 2, \mathrm{Al} 2 \mathrm{O} 3, \mathrm{FeO} 3$ e rochas calcárias onde encontramos $\mathrm{CaCO} 3$ (AMBROZEWICZ, 2012). Após a moagem e mistura destes materiais eles passam por um forno rotativo onde acontece a formação de esferas conhecidas como clínquer (NEVILLE, 2016).

De maneira simplificada podemos dizer que o clínquer é composto por C3S, $\mathrm{C} 2 \mathrm{~S}, \mathrm{C} 2 \mathrm{~A}$ e $\mathrm{C} 4 \mathrm{AF}$, também conhecidos como alita, belita, aluminato e ferrita, respectivamente. $\mathrm{Na}$ produção de compósitos cimentícios, como pastas, argamassas e concretos, a interação do cimento com água resulta no silicato de cálcio hidratado (C-S-H) e esta reação libera até $500 \mathrm{~J} / \mathrm{g}$ de cimento, por ser uma reação exotérmica (KUMAR; SINGH; SINGH, 2012). O calor é devido principalmente ao C3A (46\%), seguido pelo C3S (32\%), uma vez que o C2S (15\%) e o C4AF (7\%) liberam muito pouco calor no processo de hidratação (NEVILLE, 2016).

Ao liberar calor, sobretudo em grandes volumes de concreto, tensões de tração e compressão são introduzidas no sistema que podem apresentar patologias como microfissuras relacionadas a essas variações bruscas de volume e temperatura
(ARAÚJO, 2013). Alguns fatores podem influenciar a quantidade de calor liberada durante a hidratação do cimento entre eles a temperatura na qual a hidratação acontece, o tipo de cimento, presença de aditivos ou adições etc.

Em regiões de clima tropical, com altas temperaturas, como ocorre em quase todo o território brasileiro as altas temperaturas podem dificultar a dissipação do calor gerado durante a hidratação do concreto além de acelerar a perda de trabalhabilidade. Valin Junior et al. (2016) menciona que o calor em condições climáticas pode causar problemas ao concreto durante o período de cura e Leonhardt e Monning (1977) recomenda que durante a cura o concreto deve ser protegido contra temperaturas elevadas.

Os cimentos de alta resistência inicial, como o CP-V, demanda maior atenção neste ponto, uma vez que, ao diminuir os tempos inicial e final de pega o calor liberado fica concentrado em uma faixa mais estreita de tempo que solicita o compósito cimentício quanto as dilatações térmicas e retrações por secagem.

Por outro lado, aditivos retardadores de pega podem ser aliados retendo a trabalhabilidade, que permitem trazer um maior tempo para as etapas de transporte e aplicação dos compósitos, e retardando a hidratação que ao ocorrer de forma mais 
lenta contribui com a melhora das propriedades mecânicas e durabilidade a longo prazo (SOTOLONGO; GAYOSO; GÁLVEZ, 1993).

Alguns estudos tratam do uso da sacarose como aditivo retardador da hidratação do cimento e apresentam bons resultados com uma dosagem específica. Jumadurdiyev et al. (2005) relatam que o açúcar envolve as partículas de cimento atrasando assim a hidratação. Nas investigações de Sotolongo; Gayoso; Gálvez (1993) foi observado que, ao adicionar sacarose entre 0,1 e 0,3\% da massa de cimento, o tempo de pega foi retardado proporcionalmente a porcentagem adicionada. Ahmad; Lawan; Al-osta (AHMAD; LAWAN; AL-OSTA, 2020)(2020) obtiveram maiores incrementos nos tempos inicial e final de pega quando o teor de sacarose adicionado foi de $0,05 \%$ da massa de cimento.

Similarmente, Jamil (2010), Rattanasak; Pankhet; Chindaprasirt (2011), Kusbiantoro et al. (2013) estudaram o efeito de diversos aditivos no concreto, concluíram que a sacarose aumentou eficientemente o tempo de pega, o tempo útil de trabalhabilidade e, consequentemente, gerando menos desperdícios. Esta propriedade pode ser explicada pelo fato de a sacarose reduzir o acesso à água pelas partículas do cimento Portland, ou seja, reduz a taxa de hidratação, atrasando a formação do silicato de cálcio hidratado (C-SH) (ASSI; DEAVER; ZIEHL, 2018).

Uma fonte de sacarose pouco explorada é a vinhaça, que é um subproduto da indústria sucroalcooleira produzida em grandes volumes, o que o torna bastante atrativo nessa aplicação. Desta forma, neste trabalho estudou-se o efeito da vinhaça em pastas de cimento em estado fresco, medindo-se o tempo de pega inicial e final, em diferentes percentuais de substituição da água por vinhaça. Adicionalmente, realizouse o estudo da liberação de calor durante o processo de hidratação das diferentes pastas.

\section{METODOLOGIA}

\subsection{Materiais}

O cimento CP V-ARI foi utilizado em todas as misturas, este cimento apresenta mais de $95 \%$ de clínquer na composição. Como agentes hidratantes foi utilizada água destilada e a vinhaça in natura, disponibilizada por uma usina localizada na cidade de Osvaldo Cruz-SP.

\subsection{Métodos}

O teor de sacarose da vinhaça utilizada foi medida pelo método titulométrico por oxirredução de Eynon-Lane (IAL, 2008), obtendo-se os açúcares redutores totais (ART) e os açúcares solúveis totais (AST). O teor de sacarose (S) foi obtido de acordo com (LEME FILHO, 2005).

$$
\mathrm{S}=(\mathrm{ART}-\mathrm{AR}) * 0,95
$$

Foram elaboradas 4 pastas diferentes, compostas por cimento, água e vinhaça em diferentes percentuais, de acordo com a tabela 1.

Tabela 1. Traços das 4 pastas estudadas

\begin{tabular}{cccc}
\hline Traço & $\begin{array}{c}\text { Cimento } \\
\text { (g) }\end{array}$ & $\begin{array}{c}\text { Água } \\
\text { (\%) }\end{array}$ & $\begin{array}{c}\text { Vinhaça } \\
\text { (\%) }\end{array}$ \\
\hline $\begin{array}{c}\text { Referência (0\% } \\
\text { Vinhaça) }\end{array}$ & 500 & 100 & - \\
25\% Vinhaça & 500 & 75 & 25 \\
50\% Vinhaça & 500 & 50 & 50 \\
100\% Vinhaça & 500 & 0 & 100 \\
\hline
\end{tabular}

A quantidade de líquido nas pastas foi determinada através das recomendações da NBR 16606 (ABNT, 2018a) que estabelece o método de determinação da consistência normal $(6 \pm 1 \mathrm{~mm})$ da pasta de cimento Portland. Todas as amostras foram avaliadas a fim de determinar a influência da vinhaça na consistência das pastas.

O tempo inicial e final de pega das pastas de cimento foram determinadas com o equipamento de Vicat, conforme NBR 16607 (ABNT, 2018b). O tempo inicial de pega foi determinado pelo intervalo de tempo entre a mistura da água/vinhaça com o cimento e o momento em que penetração da agulha na pasta dista $4 \pm 1 \mathrm{~mm}$ da base. Já 
o tempo final de pega é marcado pelo intervalo de tempo transcorrido da adição de água/vinhaça ao cimento até o momento em que agulha penetra apenas $0,5 \mathrm{~mm}$ na superfície da pasta.

Buscando relacionar as variações de temperatura provenientes das reações exotérmicas de hidratação do cimento e os tempos de início e fim de pega determinados no ensaio com Vicat utilizou-se metodologia baseada no ensaio de calorimetria semiadiabática.

Para o ensaio de calorimetria semiadiabática, foi desenvolvido e fabricado um aparato para aferir o comportamento da temperatura nas pastas de cimento. Confeccionou-se uma estrutura cilíndrica de poliestireno expansível (EPS) com dimensões de $16 \mathrm{~cm}$ de altura, $8,2 \mathrm{~cm}$ de diâmetro com $15 \mathrm{~mm}$ de espessura, conforme mostra a Figura 1. Uma abertura central com $5 \mathrm{~mm}$ de diâmetro, foi aberta na parte superior para inserir o termostato, que ficou durante todo o tempo de ensaio em contato com a pasta. A abertura foi vedada para que não houvesse trocas de temperatura com o ambiente externo. Dentro do cilindro de EPS, ainda foi fixado um segundo cilindro de acetato, com $15 \mathrm{~cm}$ de altura e diâmetro de $6 \mathrm{~cm}$ para impedir o contato direto com a parede de EPS. Para proteção do aparelho de medição o termostato foi revestido com um fino tubo de polímero, com espessura $\sim 0,5 \mathrm{~mm}$. A pasta de cimento foi depositada no interior do cilindro de acetato, o termostato posicionado no núcleo da pasta, e todo o aparato foi lacrado para que não houvesse influência da temperatura externa na medição do calor liberado pela pasta. A variação de temperatura foi monitorada utilizando uma unidade de troca e registro de dados da Keysight, modelo 34970A assistida pelo do software BenchLink Data Logger 3 . O intervalo de tempo entre o contato do líquido como cimento e o início da medida da temperatura foi padronizado em 10 minutos.
Figura 1. Esquema da estrutura utilizada para o ensaio calorimétrico

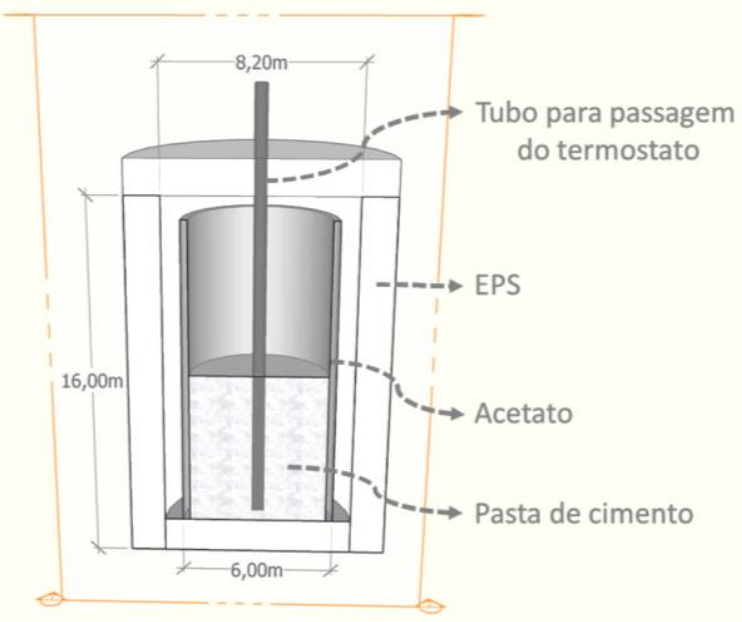

Fonte: Os autores.

\section{RESULTADOS E DISCUSSÕES}

\subsection{Açucares na vinhaça}

Os teores de açúcares foram obtidos em triplicata, pelo método titulométrico por oxirredução de Eynon-Lane. Após a conversão usando a equação 1 , o teor de sacarose na vinhaça foi de $0,650 \pm 0,003 \mathrm{~g}$ no volume de $100 \mathrm{ml}$ de vinhaça. A Tabela 2 mostra o teor de sacarose em relação à massa de cimento. Foi obtido levando-se em consideração o valor presente na vinhaça, a massa de cimento (500 g) e o fator a/c $(0,34)$.

Tabela 2. Percentuais de sacarose em relação ao cimento dos traços

\begin{tabular}{lc}
\hline \multicolumn{1}{c}{ Traço } & $\begin{array}{c}\% \\
\text { sacarose }\end{array}$ \\
\hline Referência & 0 \\
$25 \%$ vinhaça & 0,05 \\
$50 \%$ vinhaça & 0,10 \\
$100 \%$ vinhaça & 0,20 \\
\hline
\end{tabular}

\subsection{Consistência}

Como resultado do ensaio para determinação da consistência normal obteve-se o fator a/c 0,34 para a pasta de referência (água). Posteriormente, utilizou-se a mistura de água e vinhaça, enquanto produto de hidratação, nas quantidades indicadas na Tabela 1. Verificou-se que, em 
todos percentuais testados, a vinhaça não influenciava a consistência da pasta o que motivou o uso do mesmo fator a/c em todas as pastas.

\subsection{Determinação do tempo de pega}

A Figura 2 mostra os resultados de média e desvio padrão dos tempos de pega inicial e final das pastas obtidos segundo a norma NBR 16607 (ABNT, 2018b).

Figura 2. Média e desvio padrão do tempo de pega inicial e final das pastas

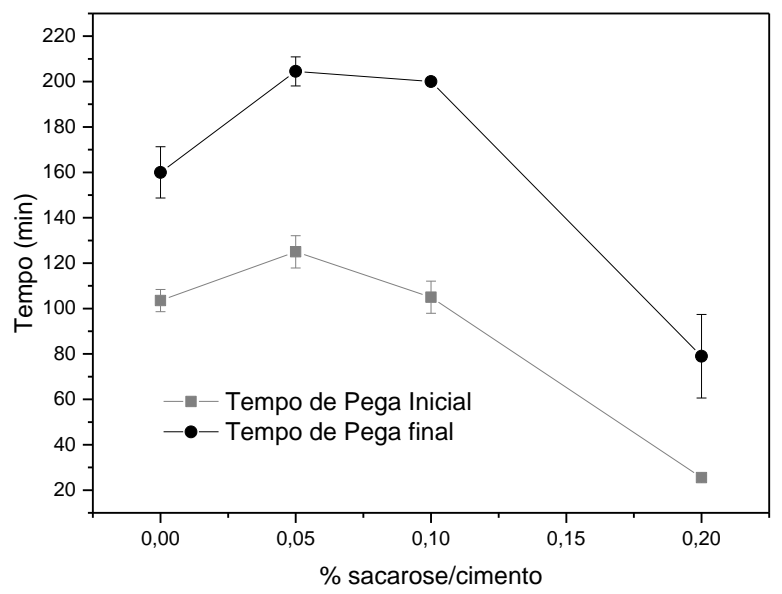

Fonte: Os autores.

Podemos observar que a substituição de $25 \%$ de água por vinhaça acarreta aumento aproximado de $21 \%$ e $28 \%$ nos tempos inicial e final de pega. $O$ atraso no tempo de pega inicial deve-se à adsorção de material orgânico no hidróxido de cálcio, reduzindo a hidratação inicial do cimento. Os maiores tempos de pega representam grande benefício, pois além do aumento do período de trabalhabilidade, pode levar à diminuição das patologias construtivas, uma vez que a hidratação mais lenta previne microfissuras relacionadas às variações bruscas de temperatura. Adicionalmente, a literatura relata que a quantidade de $0,05 \%$ de açúcar em relação ao cimento leva à um aumento da resistência à compressão (AHMAD; LAWAN; AL-OSTA, 2020).

A substituição de $50 \%$ resultou em comportamento semelhante ao obtido com a água, no tempo de pega inicial. Porém um com atraso no tempo de pega final, semelhante ao obtido com $25 \%$ de substituição. Esse resultado está em concordância com o descrito por Ahmad; Lawan; Al-osta (2020), ao utilizar sacarose como aditivo. Desta forma, pode-se obter um ganho em relação à redução de patologias, conforme mencionado anteriormente. Além disso, representa uma considerável forma de redução do consumo de água tratada na construção civil, trazendo vantagens sob ponto de vista ambiental. Já a substituição total de água por vinhaça, acarreta grandes alterações em relação ao tempo de pega e outros estudos podem ser conduzidos para viabilizar o seu uso.

\subsection{Ensaio de calorimetria}

A Figura 3 mostra os resultados da variação térmica durante $o$ processo de hidratação do cimento das pastas referência e com $25 \%$ e $50 \%$ de substituição de água por vinhaça.

Figura 3. Variação de temperatura durante a hidratação do cimento da amostra referência e com 25 e $50 \%$ de substituição de água por vinhaça

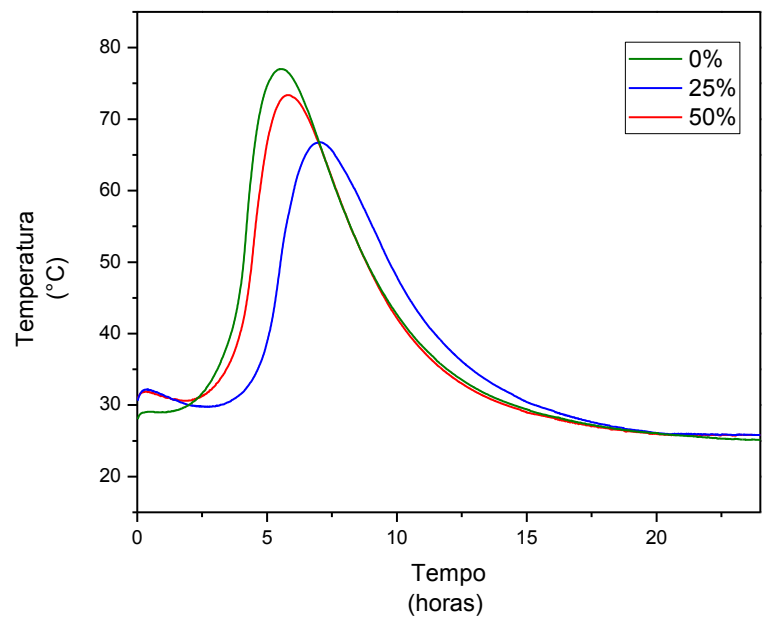

Fonte: Os autores.

O início da curva refere-se ao estágio onde a pasta começa a deixar o estado plástico e por isso as etapas de mistura, transporte e aplicação devem estar finalizadas. Por estes motivos é muito importante se conhecer esse período que representa o tempo inicial de pega. Nele as pastas começam a enrijecer e a liberação de 
calor aumenta (VIECILI; HASTENPFLUG; GIRARDI, 2018), como pode ser visto na Figura 3. O calor produzido deve-se principalmente à hidratação do C3S. O C3S compõe entre $50 \%$ e $65 \%$ dos cimentos sendo responsável pelo endurecimento rápido e pela resistência inicial dos compósitos, o que causa alta liberação de calor de hidratação (AMBROZEWICZ, 2012)

$O$ resultado da variação de temperatura está em concordância ao que foi obtido em relação ao tempo de pega (Figura 2). A curva respectiva à substituição de $25 \%$ de água está deslocada, em relação ao traço de referência (0\%). Da mesma forma, o traço com $50 \%$ de substituição tem um comportamento semelhante ao de referência (0\%). A curva com pico inferior do traço $25 \%$ mostra também uma menor variação térmica, o que pode contribuir para melhoria do material, em termos de prevenção da formação de microfissuras.

A pasta elaborada com $100 \%$ de substituição de água por vinhaça tem seu tempo de pega muito rápido, o que inviabilizou o experimento.

\section{CONSIDERAÇÕES FINAIS}

O uso da vinhaça em substituição à água na composição de matrizes cimentícias é viável. O percentual de $25 \%$ de substituição resulta no atraso do tempo de pega inicial e final, e uma menor variação térmica. O percentual de $50 \%$ resulta em comportamento semelhante ao traço referência, em termos de propriedade em estado fresco. Já a substituição total acarretou a antecipação dos tempos de pega o que pode ser prejudicial a produtividade uma vez que o tempo de disponível entre o preparo do compósito e a aplicação estaria reduzido.

\section{AGRADECIMENTOS}

Os autores agradecem a Coordenação de Aperfeiçoamento Pessoal de Nível Superior (CAPES) e a Associação Prudentina de Educação e Cultura (APEC) pela concessão de subsídios à pesquisa; Ao Programa de Pós-
Graduação em Meio Ambiente e Desenvolvimento Regional (PPGMADRE) da Universidade do Oeste Paulista (UNOESTE) e ao Instituto Federal de São Paulo (IFSP), campus Presidente Epitácio.

\section{REFERÊNCIAS}

ABNT. NBR 16606: Cimento Portland Determinação da pasta de consistência normal. Rio de Janeiro: ABNT, 2018a.

ABNT. NBR 16607: Cimento Portland Determinação dos tempos de pega. Rio de Janeiro: ABNT, 2018b.

AHMAD, S.; LAWAN, A.; AL-OSTA, M. Effect of sugar dosage on setting time, microstructure and strength of Type I and Type V Portland cements. Case Studies in Construction Materials, v. 13, p. e00364, dez. 2020. https://doi.org/10.1016/j.cscm.2020.e00364

AMBROZEWICZ, P. H. L. Materiais de Construção Civil: Normas, especificações, aplicação e ensaios de laboratório. São Paulo: PINI, 2012.

ARAÚJO, J. M. Tensões térmicas em blocos de concreto armado. Teoria e Prática na Engenharia Civil, v. 22, p. 1-14, 2013.

ASSI, L. N.; DEAVER, E. (EDDIE); ZIEHL, P. Using sucrose for improvement of initial and final setting times of silica fume-based activating solution of fly ash geopolymer concrete. Construction and Building Materials, v. 191, p. 47-55, dez. 2018. https://doi.org/10.1016/j.conbuildmat.2018. $\underline{09.199}$

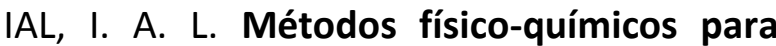
análise de alimentos. São Paulo, Brasil: [s.n.]. v. 4

JAMIL, N. N. B. The effect of natural retarder on geopolymer concrete with different curing regime. [S.I.]: Universiti Teknologi Petronas, 2010. 
JUMADURDIYEV, A. et al. The utilization of beet molasses as a retarding and waterreducing admixture for concrete. Cement and Concrete Research, v. 35, n. 5, p. 874882, maio 2005. https://doi.org/10.1016/i.cemconres.2004.04 $\underline{.036}$

KUMAR, M.; SINGH, S. K.; SINGH, N. P. Heat evolution during the hydration of Portland cement in the presence of fly ash, calcium hydroxide and super plasticizer. Thermochimica Acta, v. 548, p. 27-32, nov. 2012.

https://doi.org/10.1016/i.tca.2012.08.028

KUSBIANTORO, A. et al. Development of Sucrose and Citric Acid as the Natural based Admixture for Fly Ash based Geopolymer. Procedia Environmental Sciences, v. 17, p. 596-602, 2013. https://doi.org/10.1016/i.proenv.2013.02.07 $\underline{5}$

LEME FILHO, J. R. A. Estudo comparativo dos métodos de determinação e de estimativa dos teores de fibra e de açúcares redutores em cana-de-açúcar (Saccharum spp.). Piracicaba: Universidade de São Paulo, 2005.

LEONHARDT, F.; MONNIG, E. Construções de concreto: princípios básicos do dimensionamento de estruturas de concreto armado. Rio de Janeiro: Interciência, 1977. v.1

NEVILLE, A. M. Propriedades do concreto. Porto Alegre: Bookman, 2016.

RATTANASAK, U.; PANKHET, K.; CHINDAPRASIRT, P. Effect of chemical admixtures on properties of high-calcium fly ash geopolymer. International Journal of Minerals, Metallurgy, and Materials, v. 18, n. 3, p. 364-369, 29 jun. 2011. https://doi.org/10.1007/s12613-011-0448-3

SOTOLONGO, R.; GAYOSO, R.; GÁLVEZ, M. Contribución al estudio de la sacarosa como aditivo retardador de la hidratación del cemento. Materiales de Construcción, v. 43, n. 230, p. 37-39, 30 jun. 1993. https://doi.org/10.3989/mc.1993.v43.i230.68 $\underline{2}$

VALIN JR, M. DE O. et al. Influência de diferentes climas urbanos na qualidade do concreto. Revista Engenharia e Construção Civil, v. 3, p. 40-54, 2016.

VIECILI, M. J. C.; HASTENPFLUG, D.; GIRARDI, R. Comparativo entre o teste de Vicat e a calorimetria semi-adiabática para determinação do tempo de início e fim de pega em cimentos Portland. Matéria (Rio de Janeiro), v. 23, n. 3, 18 out. 2018. https://doi.org/10.1590/s1517$\underline{707620180003.0506}$ 